\title{
COST AND IMPACT OF SELF-REPORT AND TELEMONITORING ON HEART FAILURE READMISSION: A CASE REPORT
}

doi:10.1136/heartjnl-2012-302920o.26

${ }^{1}$ Nan Li, ${ }^{2}$ Xinru Dong, ${ }^{1}$ Bernard Cheung, ${ }^{1}$ Bernard Cheung. ${ }^{1}$ Department of Medicine, University of Hong Kong, Hong Kong, China; ${ }^{2}$ As-A Class, Guanghua College, Fudan Campus, Shanghai, China

Objectives Chronic heart failure is a progressive pathological process with frequent rehospitalisation, low survival rate and high medical cost. Early detection of signs of decompensation events is critical for reducing hospital readmission.

Methods An 83-year-old man with chronic heart failure presented early signs of decompensation four times since 2010 and were alerted to the HF specialist through the combination of self-report and telemonitoring. The patient's symptoms were treated in time and hospital readmission was avoided. The cost of the combination in the first year was 230.0 USD, which was $85 \%$ of the cost of regular tests (271.0 USD), 30\% of the cost of hospital per stay (783.7 USD), and $15 \%$ of the cost of the general medications per year (2351.0 USD). From the second year, the cost of the combination (89.9 USD) declined to $33 \%$ of the regular tests, $11 \%$ of the cost of hospital per stay, and $6 \%$ of the cost of general medications per year.

Results An 83-year-old man with chronic heart failure presented early signs of decompensation four times since 2010 and were alerted to the HF specialist through the combination of self-report and telemonitoring. The patient's symptoms were treated in time and hospital readmission was avoided. The cost of the combination in the first year was 230.0 USD, which was $85 \%$ of the cost of regular tests (271.0 USD), 30\% of the cost of hospital per stay (783.7 USD), and $15 \%$ of the cost of the general medications per year (2351.0 USD). From the second year, the cost of the combination (89.9 USD) declined to $33 \%$ of the regular tests, $11 \%$ of the cost of hospital per stay, and $6 \%$ of the cost of general medications per year. 


\section{ABSTRACTS}

Conclusions The combination of self-report and telemonitoring is an inexpensive but effective measure to improve the early detection of signs of decompensation event so as to allow prompt treatment and, reduce the high cost of hospital readmission in the management of chronic heart failure. 